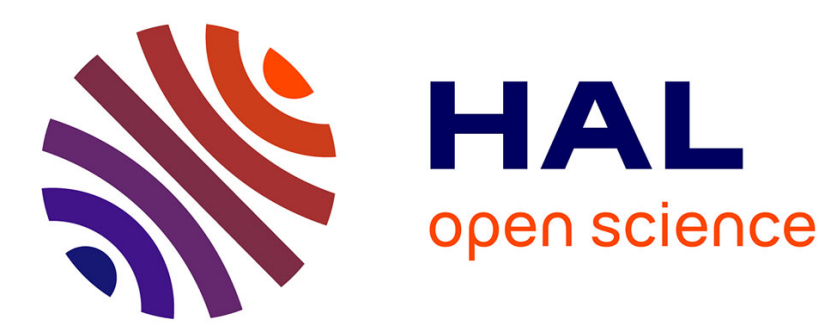

\title{
A Volume Integral Formulation Based on Facet Elements for Nonlinear Magnetostatic Problems
}

Vinh Le Van, Gérard Meunier, Olivier Chadebec, Jean-Michel Guichon

\section{To cite this version:}

Vinh Le Van, Gérard Meunier, Olivier Chadebec, Jean-Michel Guichon. A Volume Integral Formulation Based on Facet Elements for Nonlinear Magnetostatic Problems. IEEE Transactions on Magnetics, 2015, 51 (7), 10.1109/TMAG.2015.2389197 . hal-01177829

\section{HAL Id: hal-01177829 \\ https://hal.science/hal-01177829}

Submitted on 24 Nov 2020

HAL is a multi-disciplinary open access archive for the deposit and dissemination of scientific research documents, whether they are published or not. The documents may come from teaching and research institutions in France or abroad, or from public or private research centers.
L'archive ouverte pluridisciplinaire HAL, est destinée au dépôt et à la diffusion de documents scientifiques de niveau recherche, publiés ou non, émanant des établissements d'enseignement et de recherche français ou étrangers, des laboratoires publics ou privés. 


\title{
A Volume Integral Formulation Based on Facet Elements for Nonlinear Magnetostatic Problems
}

\author{
Vinh Le-Van, Gérard Meunier, Olivier Chadebec and Jean-Michel Guichon \\ Univ. Grenoble Alpes, G2Elab, F-38000 Grenoble, France \\ CNRS, G2Elab, F-38000 Grenoble, France
}

\begin{abstract}
This paper presents a novel and useful 3D nonlinear magnetostatic integral formulation for volume integral method. Like every other integral formulation, its main advantage is that it does not require air region mesh, only ferromagnetic regions being discretized. The formulation is based on magnetic flux density interpolation on facet elements. Special care is taken in order to accurately compute the singularity of Green's kernel. The application of an equivalent circuit approach allows preserving the solenoidality of magnetic induction. It is shown that the formulation is very accurate even if it is associated with coarse meshes. Thus, computation time can be very competitive. Computed results for the TEAM Workshop problem 13 and for a multiply-connected regions case-test are reported.
\end{abstract}

Index Terms - facet elements, nonlinear magnetostatics, volume integral method.

\section{INTRODUCTION}

$\mathrm{V}$ olume Integral Method (VIM) is known to be a powerful approach to model magnetostatic problems like actuator devices. Several academic codes like GFUN [1], GFUNET/CORAL [2], RADIA [3] or CALMAG3D [4] developed since the 1970s have already shown the reliability of the approach. The main advantage of VIM is that neither free space mesh nor boundary conditions are required. Using VIM, only active regions have to be discretized into elementary elements. This allows in general resolving accurately magnetostatic problems with very coarse meshes. VIM has a renewed interest in the last decade by the development of matrix compression algorithms such as the FMM (Fast Multipole Method) [5], the HCA (Hybrid Cross Approximation) [6], which improve efficiently both storage memory and resolution time of fully populated matrix systems. Moreover, a Nyström discretization technique [7] [8] allows incorporating both higher-order meshes and higherorder basis functions in order to more accurately solve the magnetostatic integral equation. As a result, VIM is an interesting alternative to classical finite element method (FEM) methods in some situations today.

The main idea of the magnetostatic VIM approach is that the magnetic field is created by the inductor sources and all ferromagnetic elements defined in the problem. With a point matching technique or a Galerkin's projection, which ensures the validity of the materials constitutive laws, a matrix system is obtained. It remains to solve this matrix system to get quantity values on the mesh of the ferromagnetic part and then to compute the magnetic field everywhere. Force or flux can also be easily computed.

Different kinds of magnetostatic volume integral formulations based on different quantities interpolations have already been presented in the literature. The oldest and also most popular one is based on uniform magnetization on each element. This formulation is usually known as Magnetic Moment Method (MMM) [9] [10] [11] and is certainly the simplest to implement. The MMM is capable of yielding accurate results for simple geometric devices [10] but can suffer from the "looping pattern" as mentioned in [12]. If the susceptibility of the material is high, the associated matrix system becomes singular and the solution contains some spurious modes, decreasing its accuracy. This is why better formations associated with more reliable field interpolation basis are preferred. Many authors have proposed volume integral formulations used nodal and edge elements interpolations. The formulation based on nodal elements and magnetic scalar potential $\varphi$ [13] [14] [15] significantly improves the accuracy of VIM in comparing with MMM. The $\varphi$-nodal formulation allows for minimizing the number of unknowns. However, it requires a particular cutting technique to resolve multiple-connected regions problems. Moreover, an additional pre-resolution is needed in order to compute the scalar potential $\varphi_{0}$ in the presence of coils. The $\mathbf{H}$-edge formulation that is based on the interpolation of magnetic fields with edge elements also yields accurate results [2] [16]. It does not require a source pre-resolution and avoids the cutting technique for multiple-connected regions problems by using an edge-tree technique.

Regarding to the nonlinear resolution, all three formulations MMM, $\varphi$-nodal, and $\mathbf{H}$-edge have poor convergence rates due to the use $\mathbf{B}(\mathbf{H})$ or $\mathbf{M}(\mathbf{H})$ curve. If the Newton-Raphson (NR) method is used, relaxation has to be very often required in order to achieve convergence. Yet, the determining of optimal constant relaxation factor involves additional studies, such as by using an energy minimum principle [17]. Different from mentioned formulations above, we propose in this paper a novel magnetostatic VIM formulation based on facet elements of first order associated with magnetic flux density, called Bfacet formulation. The degrees of freedom are associated with the normal component of magnetic flux density on facets of the mesh. The divergence-free magnetic-field condition is achieved by using an equivalent circuit approach. We will demonstrate that the formulation leads to the use of a $\mathbf{H}(\mathbf{B})$ 
curve during the nonlinear resolution, and as a result, good NR convergence rates can be easy obtained without any relaxation.

Some papers have also proposed the use of facet elements interpolation for magnetostatics [18] [19]. However, in [18] Vishnevsky et al. (2002) suggested the interpolation of magnetization on facet elements. This approach can be suitable for linear cases but should not be applied generically for nonlinear problems, because the normal component of the magnetization across facets could not be considered continuous, unlike one of the magnetic flux. In [19], although Balasubramanian et al. (2002) used the B-facet interpolation, the solenoidality of magnetic induction is not strictly satisfied. And this should lead to an inaccuracy of the solution in some situations. In our formulation, the B-facet interpolation is used as well but the solenoidality of magnetic induction is strongly imposed thanks to the use of an equivalent circuit approach. Moreover, the singularity the Green's function is computed accurately by using an analytical formula. A Nyström discretization technique [7] [8] could be envisaged in order to incorporate both higher-order meshes and higher-order basis functions.

This paper is organized in the following sections. Section II is dedicated to magnetostatic integral equation, Section III presents properties of facet element shape-function. Section IV describes the new volume integral formulation. Numerical examples are shown in the Section V and Section VI is the conclusion.

\section{BASIS EQUATIONS}

The governing equations for magnetostatic problem are

$$
\begin{aligned}
& \operatorname{div} \mathbf{B}=0, \\
& \operatorname{rot} \mathbf{H}=\mathbf{J},
\end{aligned}
$$

where $\mathbf{B}, \mathbf{H}, \mathbf{J}$ are the magnetic flux density, the magnetic field intensity, the current density respectively. Without any permanent magnetization, the constitutive equation is:

$$
\begin{gathered}
\mathbf{H}=v(\mathbf{B}) \mathbf{B}, \\
\mathbf{M}=v_{0} \mathbf{B}-v(\mathbf{B}) \mathbf{B},
\end{gathered}
$$

where $v_{0}$ and $v(\mathbf{B})$ denotes the reluctivity of vacuum and material respectively, $\mathbf{M}$ is the magnetization.

The magnetic field $\mathbf{H}$ can be decomposed into the sum of two terms: $\mathbf{H}_{0}$, the source field created by source currents and $\mathbf{H}_{r}$, the reduced field created by ferromagnetic materials. Based on the Maxwell-Ampere's equation (2), it can be shown that $\mathbf{H}_{\mathrm{r}}$ derives from a reduced magnetic scalar potential $\varphi_{r}$. Hence, we have

$$
\mathbf{H}=\mathbf{H}_{0}-\operatorname{grad} \varphi_{r}
$$

The scalar potential $\varphi_{r}$ is determined by

$$
\varphi_{r}=\frac{1}{4 \pi} \int_{\Omega} \mathbf{M} \cdot \operatorname{grad}\left(\frac{1}{r}\right) \mathrm{d} \Omega,
$$

where $r$ is the distance between the observation and integration points and $\Omega$ is magnetic region. From (3) and (5) we get

$$
v(\mathbf{B}) \mathbf{B}+\operatorname{grad} \varphi_{r}=\mathbf{H}_{0} .
$$

\section{FACET SHAPE FUNCTION PROPERTIES}

Shape functions of facet elements can be generated from nodal shape functions. Let facet $i$ belong to a tetrahedral element $E$. If facet $i$ is defined by a set of three nodes $\{a, b, c\}$. The first-order shape-function can be written as

$$
\mathbf{w}_{i}=2\left(\lambda_{a} \nabla \lambda_{b} \times \nabla \lambda_{c}+\lambda_{b} \nabla \lambda_{c} \times \nabla \lambda_{a}+\lambda_{c} \nabla \lambda_{a} \times \nabla \lambda_{b}\right),
$$

where $\lambda$ is the classical node shape function.

The flux of $\mathbf{w}_{i}$ across facet $i$ is equal to one and vanishes across other faces. If facet $i$ has an inner orientation, we have

$$
\begin{gathered}
\mathbf{w}_{i} \cdot \mathbf{n}_{i}=\frac{1}{\mathrm{~S}_{\mathrm{i}}}, \\
\operatorname{div} \mathbf{w}_{i}=\frac{1}{\mathrm{~V}_{\mathrm{E}}},
\end{gathered}
$$

where $\mathbf{n}_{i}$ is the ingoing normal vector, $\mathrm{S}_{\mathrm{i}}$ the surface of $i$ and $\mathrm{V}_{\mathrm{E}}$ the volume of $E$. These properties remain identical, but with opposite sign if the facet $i$ has an outer orientation. Equations (9.a), (9.b) are still valid for hexahedral elements and therefore the all formula developed hereafter in this paper are also valid for hexahedral elements mesh. Details about facet elements properties can be found in [20].

\section{FORMULATIONS}

\section{A. Facet Elements Discretization}

The ferromagnetic body $\Omega$ is subdivided into $N$ elementary volume elements. Let $\Gamma$ be the boundary of $\Omega ; N_{f}$ be the number of facet elements on $\Omega ; N_{b}$ the number of facet elements on the boundary $\Gamma$.

Vector $\mathbf{B}$ can be approximated by facet elements interpolation of first order as

$$
\mathbf{B}=\sum_{j=1}^{N_{f}} \mathbf{w}_{j} \Phi_{j},
$$

where $\Phi_{j}$ denotes the magnetic flux across the facet $j$.

Projecting (7) on $\Omega$ by the Galerkin method with facet shape-function $\mathbf{w}_{i}$, we get

$$
\int_{\Omega} v(\mathbf{B}) \mathbf{B}+\int_{\Omega} \mathbf{w}_{i} \cdot \operatorname{grad} \varphi_{r} \mathrm{~d} \Omega=\int_{\Omega} \mathbf{w}_{i} \cdot \mathbf{H}_{0} \mathrm{~d} \Omega .
$$


Then according to (10), we have:

$$
\sum_{j=1}^{N_{f}}\left(\int_{\Omega} \mathbf{w}_{i} \cdot v(\mathbf{B}) \mathbf{w}_{j} \mathrm{~d} \Omega\right) \Phi_{j}+\int_{\Omega} \mathbf{w}_{i} \cdot \operatorname{grad} \varphi_{r} \mathrm{~d} \Omega=\int_{\Omega} \mathbf{w}_{i} \cdot \mathbf{H}_{0} \mathrm{~d} \Omega
$$

In matrix-vector form, (12) is rewritten as

$$
\mathbf{R} \Phi+\int_{\Omega} \mathbf{w}_{i} \cdot \operatorname{grad} \varphi_{r} \mathrm{~d} \Omega=F
$$

where $\mathbf{R}$ denotes a finite-element matrix, $\Phi$ is the vector of flux through facets, and $F$ is a vector corresponding to the source-field term. Expressions of $\mathbf{R}$ and $F$ are

$$
\begin{aligned}
& \mathbf{R}_{i, j}=\int_{\Omega} \mathbf{w}_{i} \cdot v(\mathbf{B}) \mathbf{w}_{j} \mathrm{~d} \Omega \\
& F_{i}=\int_{\Omega} \mathbf{w}_{i} \cdot \mathbf{H}_{0} \mathrm{~d} \Omega
\end{aligned}
$$

Applying the divergence theorem, we can write the integral term in (13) as

$$
\mathbf{I}=\int_{\Omega} \mathbf{w}_{i} \cdot \operatorname{grad} \varphi_{r} \mathrm{~d} \Omega=\int_{\Gamma} \varphi_{r} \mathbf{w}_{i} \cdot \mathbf{n}_{i} \mathrm{~d} \Gamma-\int_{\Omega} \varphi_{r} \operatorname{div} \mathbf{w}_{i} \mathrm{~d} \Omega .
$$

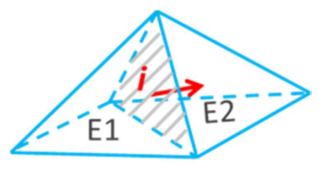

(a)

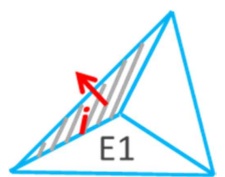

(b)
Fig.1. Facet identification. a) Inner facet. b) Border facet.

- For inner facet $i$ (Fig. 1.a), the surface integral term in (13) vanishes. So thanks to (9.b) we have

$$
\mathbf{I}=\int_{\Omega_{\mathrm{E} 2}} \frac{\varphi_{r}}{\mathrm{~V}_{\mathrm{E} 2}} \mathrm{~d} \Omega_{\mathrm{E} 2}-\int_{\Omega_{\mathrm{E} 1}} \frac{\varphi_{r}}{\mathrm{~V}_{\mathrm{E} 1}} \mathrm{~d} \Omega_{\mathrm{E} 1}=\varphi_{\mathrm{E} 2}-\varphi_{\mathrm{E} 1}=\Delta \varphi_{\mathrm{IN} i}
$$

- For outer facet $i$, i.e. located on the $\Gamma$ (Fig. 1.b), thanks to (9.a) we have

$$
\mathbf{I}=\int_{\Gamma_{i}} \frac{\varphi_{r}}{S_{\mathrm{i}}} \mathrm{d} \Gamma_{\mathrm{i}}-\int_{\Omega_{\mathrm{E} 1}} \frac{\varphi_{r}}{\mathrm{~V}_{\mathrm{E} 1}} \mathrm{~d} \Omega_{\mathrm{E} 1}=\varphi_{\mathrm{i}}-\varphi_{\mathrm{E} 1}=\Delta \varphi_{\Gamma \mathrm{i}}
$$

where $\varphi_{\mathrm{E}}, \varphi_{\mathrm{i}}$ are the average reduced scalar potentials of element $\mathrm{E}$ and facet $i$, respectively; $\Delta \varphi_{\mathrm{IN}} i$ denotes the difference of average potentials between two adjacent elements; $\Delta \varphi_{\Gamma i}$ denotes one between the element and its border facet.

In short, (13) is represented as

$$
\mathbf{R} \Phi+\left(\begin{array}{c}
\Delta \varphi_{\mathrm{IN}} \\
\Delta \varphi_{\Gamma}
\end{array}\right)=F
$$

In the next subsection an equivalent circuit approach is briefly introduced in order to develop explicitly two terms $\Delta \varphi_{\mathrm{IN}}$ and $\Delta \varphi_{\Gamma}$ in order to complete the equation system.

\section{B. Equivalent Circuit Approach}

The equivalent circuit approach has been widely used for a long time to find numerical solutions of electromagnetic field problems. The key point of the approach is to establish a network model thanks to the analogy between magnetic circuit and electric circuit. In [21] [22] and [23], network models are established by means of the FEM. In [24] and [25], authors introduce the use of networks model into the VIM.

In our approach, the equivalent-circuit-model has nodes which are associated with volume-element centers and an outer domain node $\infty$, called the infinity node. The circuit branches are either the branches through inner facet which connect centers of both adjacent elements, or the branches through boundary facet and link the center of boundary volume elements with infinity node. The Fig. 2 illustrates an example of equivalent circuit model for a 2D mesh of 6 triangle elements.

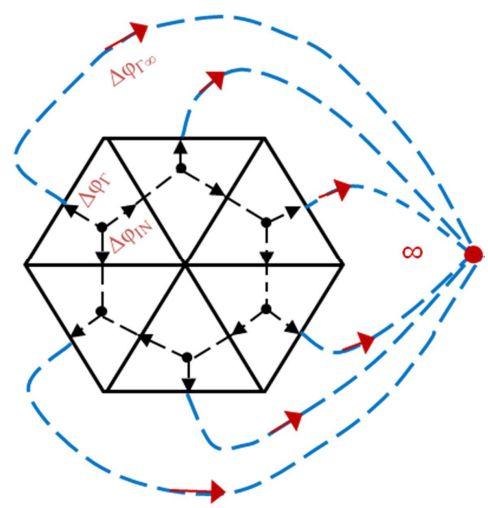

Fig.2. Equivalent circuit model for 2D mesh.

Based on this approach, equation (16) can be interpreted as follows: the matrix $\mathbf{R}$ is equivalent to reluctance network, $\Delta \varphi_{\mathrm{IN}}, \Delta \varphi_{\Gamma \text { in }}$ correspond to magnetic flux leakages and $F$ is the magneto-motive force. The aim of the next subsection is to establish the linear system to be solved.

\section{Integral Volume Formulation}

A common convention is to set the magnetic scalar potential at infinity $\left(\varphi_{\infty}\right)$ to zero. Thus, the difference of scalar potential between the infinity node and a border facet $i$ is

$$
\Delta \varphi_{\Gamma i \infty}=\varphi_{\infty}-\varphi_{\Gamma i}=-\int_{\Gamma_{\mathrm{i}}} \frac{\varphi_{r}}{S_{i}} \mathrm{~d} \Gamma_{\mathrm{i}}
$$

According to (4) and (6), $\varphi_{r}$ is expressed as 


$$
\varphi_{r}=\sum_{\Omega_{e} \in \Omega} \frac{1}{4 \pi} \int_{\Omega_{e}}\left(v_{0}-v_{\mathrm{E}}(\mathbf{B})\right) \mathbf{B} \cdot \operatorname{grad}\left(\frac{1}{r}\right) \mathrm{d} \Omega_{e},
$$

Hence, (17) is developed as

$$
\Delta \varphi_{\Gamma i \infty}=-\frac{1}{4 \pi} \int_{\Gamma_{i}} \frac{1}{S_{i}}\left[\sum_{j=1}^{N_{f}}\left(\frac{\delta v_{j}}{S_{j}} \int_{\Gamma_{j}} \frac{1}{r} \mathrm{~d} \Gamma_{j}\right) \Phi_{j}\right] \mathrm{d} \Gamma_{\mathrm{i}} .
$$

where $\delta v_{j}$ denotes difference of reluctivity either between two adjacent elements or between border element and air. In the matrix-vector form we write (19) as

$$
\Delta \varphi_{\Gamma \infty}+\mathbf{L} \Phi=0
$$

where $\mathbf{L}$ is the integral matrix corresponding to border facets and representing the magnetic flux leakage.

$$
\mathbf{L}_{i j}=\frac{\delta v_{j}}{4 \pi} \frac{1}{S_{i}} \frac{1}{S_{j}} \int_{\Gamma_{i}} \int_{\Gamma_{j}} \frac{1}{r} \mathrm{~d} \Gamma_{i} \mathrm{~d} \Gamma_{\mathrm{j}}=\delta v_{j} \mathbf{L}_{0 i j},
$$

with $i=1,2, . . N_{b}, j=1,2, . . N_{f}$. The singularity of Green's function with self-interaction in (21) can be avoided by using a shifting of Gauss's points or the well-known analytical integration. This analytical correction can be found in [26]. It must be pointed out that this numerical technique is the key point of the method in order to compute the integral of the Green's kernel associated with this formulation.

From (16) and (20), we have

$$
(\mathbf{R}+\mathbf{L}) \Phi+\Delta \varphi=F \quad \text { with } \quad \Delta \varphi=\left(\begin{array}{l}
\Delta \varphi_{\mathrm{IN}} \\
\Delta \varphi_{\Gamma \text { in }}+\Delta \varphi_{\Gamma \infty}
\end{array}\right)
$$

where $\mathbf{R}$ is a sparse finite-element-matrix and $\mathbf{L}$ is a dense integral matrix. The structures of $\mathbf{R}$ and $\mathbf{L}$ are illustrated in Fig. 3. In general, we have $\mathrm{N}_{b}<<\mathrm{N}_{f}$.

The mesh current method is then used to solve the equivalent circuit. Let $\mathbf{P}$ be an incidence matrix which links the incidence of the independent loops to the branches. Because of the fact that the vector $\Delta \varphi$ in (22) represents the difference of scalar potential between nodes of circuit including the infinity node, the Kirchhoff's voltage law imposes that

$$
\mathbf{P} \Delta \varphi=0 .
$$

Combining (22) and (23), we get

$$
\mathbf{P}[(\mathbf{R}+\mathbf{L}) \Phi]=\mathbf{P} F,
$$

By means of the mesh current method, (24) is written as

$$
\mathbf{P}(\mathbf{R}+\mathbf{L}) \mathbf{P}^{\mathrm{T}} \Phi_{\mathrm{M}}=\mathbf{P} F
$$

where $\mathbf{P}^{\mathrm{T}}$ is transpose of matrix $\mathbf{P}$ and $\Phi_{\mathrm{M}}$ the vector of mesh fluxes, $\Phi_{M}$ is determined by $\Phi_{M}=\mathbf{P} \Phi$.

An independent-loop-search algorithm proposed in [27] can be used for the determining of the incidence matrix $\mathbf{P}$.
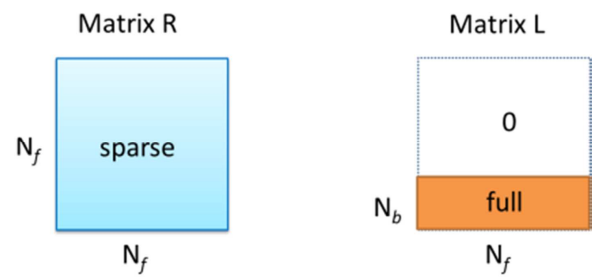

Fig. 3. Structures of $\mathbf{R}$ and $\mathbf{L}$ matrices

\section{Matrix Assembly}

For the linear case, the size of integral matrix is reduced because the permeability is constant by region. Integral interactions are then limited between elements of $\Gamma$ and all the borders/ intersections of regions.

From (21), it can be remarked that each element of integral matrix $\mathbf{L}$ is composed by two terms, one is dependent to material $\Delta \vartheta_{j}$ and the other $\mathbf{L}_{\mathbf{0} i j}$ is independent. Consequently, if the NR method is used for solving the nonlinear problem, only the sparse matrix $\mathbf{R}$ and the vector $\Delta \vartheta$ have to be recomputed iteratively. The matrix $\mathbf{L}_{\mathbf{0}}$ is computed one single time before the resolution. This saves both the memory needed and the computation time and is another key point of our approach.

\section{NUMERICAL EXAMPLES}

The proposed formulation was applied in order to solve a multiply of connected problems with nonlinear material in the first part. In the second part, it was applied to solve the TEAM Workshop Problem 13.

\section{A. Closed Magnetic Circuit Modeling}

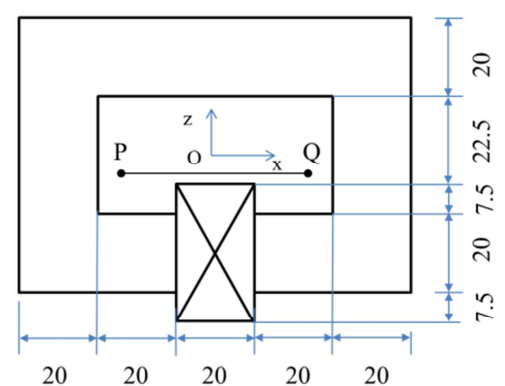

a)

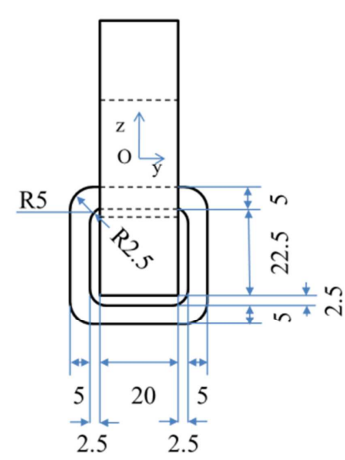

b)
Fig. 4. Geometry of magnetic circuit and coil a) Front view. b) Horizontal view

A closed magnetic circuit is excited by a DC current coil of 100 A.T. The geometry is shown on the Fig. 4 in millimeter scale. The nonlinear ferromagnetic material is specified by an arctangent law as the following equation 


$$
\mathbf{B}(\mathbf{H})=\mu_{0} \mathbf{H}+\frac{2 \mathbf{J}_{\mathrm{s}}}{\pi} \operatorname{arctg}\left(\frac{\pi\left(\mu_{r}-1\right) \mu_{0} \mathbf{H}}{2 \mathbf{J}_{\mathrm{s}}}\right),
$$

where $\mathrm{J}_{\mathrm{s}}$ the saturation magnetization is $1.0 \mathrm{~T}$, and $\mu_{r}$ the initial relative permeability is 500 .

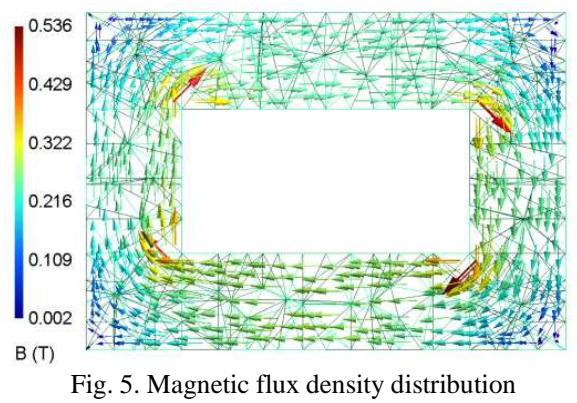

The problem was solved by the proposed formulation. A coarse mesh - 96 elements and a denser one -890 element were considered. And then, the module $|\mathbf{B}|$ were computed on the 25 points on the segment PQ in air, with $\mathrm{P}(-25,0,-5)$ and $\mathrm{Q}(25,0,-5)$. These results were compared with the reference values that had performed by the FEM by means of commercial FEM software Flux3D® with 200,000 tetrahedral elements in second order including 120,000 elements in ferromagnetic region. The coarse mesh of 96 elements gives comparable results and the results obtained with mesh are nearly coincided with the reference values.

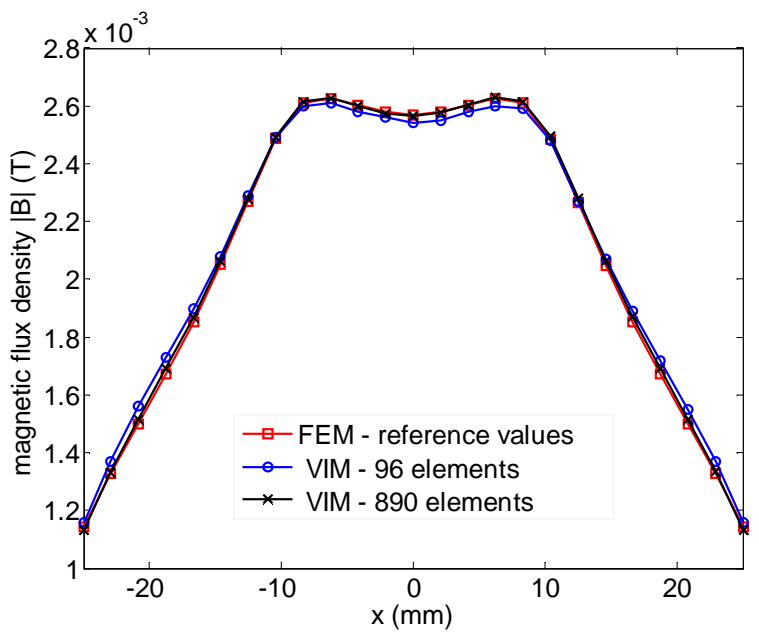

Fig. 6. Magnetic field module on the segment PQ

\section{B. TEAM Workshop Problem 13}

Benchmark problem 13 defined in the TEAM Workshop is a nonlinear magnetostatic problem [28]. An excited coil is set between two steel channels and a steel plate is inserted between the channels. The applied magnetomotive force is 1000 A.T.
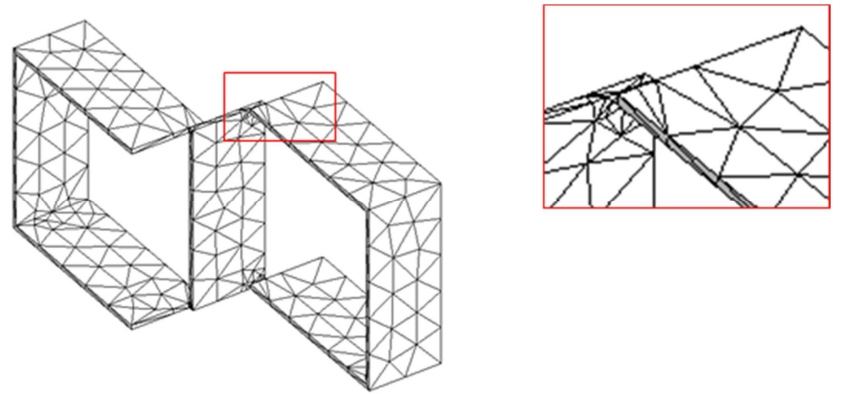

Fig. 7. Mesh of problem 13 with 1235 volume elements

The iterative solver FGMRES (Flexible Generalized Minimal Residual method) [29] is used for the solving linear system and the classic NR method is used to deal with nonlinear system. Three regular tetrahedral meshes (1235, 4,855 and 20,737 elements) were reported. In figure 7 , the mesh of 1,235 elements is presented.
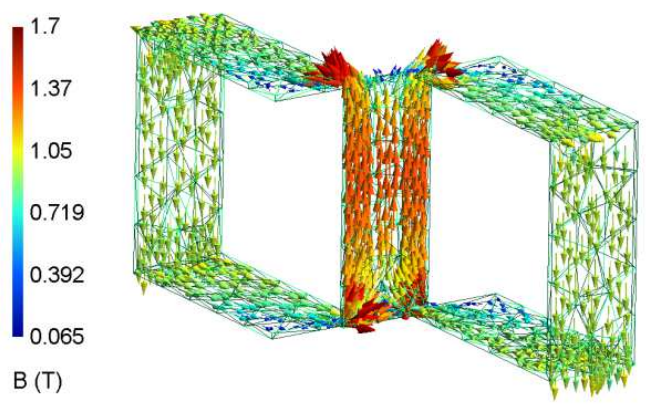

Fig. 8. Distribution of magnetic flux density vector

The distribution of magnetic flux density on the steel plates with this mesh is shown in Fig. 8. The average flux densities in the steel plate and in the air at various positions are plotted in Fig.9 and Fig. 10, respectively. Both figures indicate that the computed results are very close to the measured one. The results of spatial distributions of flux in steel plate are very close measured values, but ones in air are slightly different in some points. As shown on the Fig. 9 and Fig. 10, a coarse mesh composed of 1,235 elements can lead to accurate results. For this mesh, we needed 6.7 seconds for the NR resolution by using a computer of CPU 3.04 GHz and 1.1 GB of RAM. The convergence of the solution was obtained in five steps without any relaxation. The absolute stopping criterion of NR was set $1 \mathrm{E}-6$. For the mesh of 4,855 elements, the number of iterations was always five and the time of resolution 30,48 seconds.

The curves with 4,855 elements and 20,737 elements are superposed and very close to the measured values. Therefore, it can be concluded that the convergence was reached for a mesh of 4,855 elements. Compared to meshes used in [28], this mesh is still very coarse. The result of TEAM problem 13 validates the efficiency and accuracy of the method to analyze nonlinear models. 


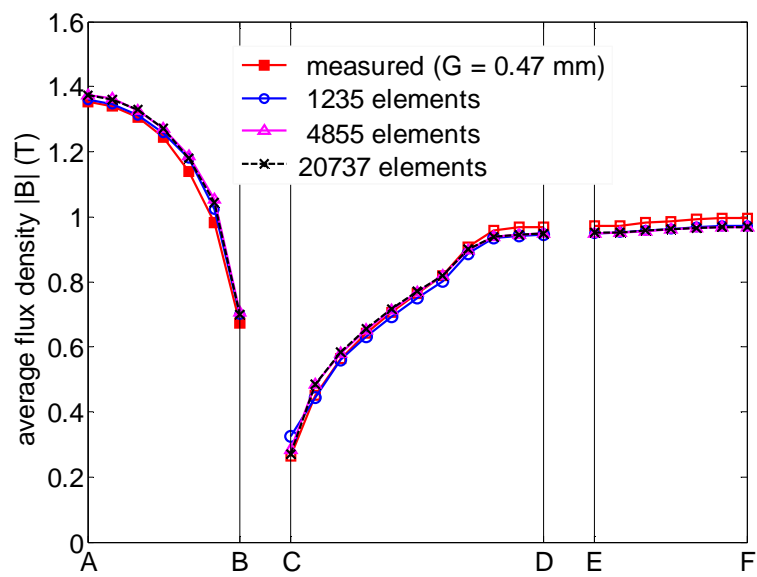

Fig. 9. Spatial distributions of average flux density in steel plate

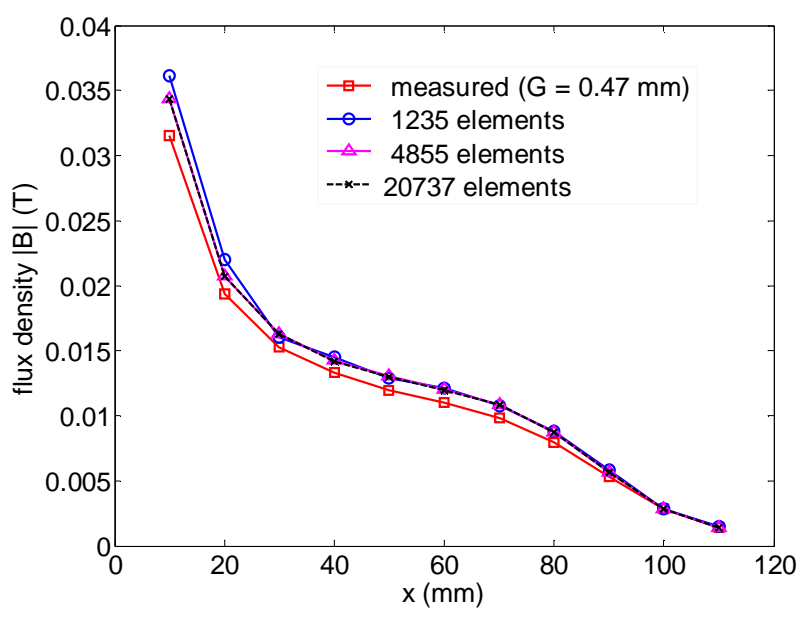

Fig. 10. Spatial distributions of flux density in air

For large problems, a fast algorithm such as the FMM, ACA (Adaptive Cross Approximation) [30] or HCA can be applied in order to save the memory and computation time. Information about the implementation of FMM and ACA for the magnetostatic VIM can be found in [19] [31] and [11] [32], respectively.

\section{CONCLUSION}

A volume integral formulation based on facet element interpolation and magnetic flux has been developed for nonlinear magnetostatic problems. The key features of the formulation were that the imposition of solenoidality of magnetic induction by using an equivalent circuit approach and the accuracy of Green's function computation. The multiply-connected-regions problem was solved without any cutting surface. The classic NR method was used to solve nonlinear magnetostatic problems. The numerical results of a multiply-connected regions case-test and of the TEAM Workshop Problem 13 demonstrated the strength, the accuracy and the efficiency of this new approach. These encouraging results inspire the use of VIM in order to deal with complex electromagnetic devices. For the further works, a development of the formulation for the resolution of magnetic thin shell as well as a coupling with eddy current formulation using facet elements for the solving of electromagnetic problems should be envisaged.

\section{REFERENCES}

[1] M. J. Newman, C. W. Trowbridge, and L. R. Turner, "GFUN: an interactive program as an aid to magnet design", Proc. 4th Int. Conf. Magnet Technology, Brookhaven National Laboratory, Upton, NY, pp. 617-626, 1972.

[2] L. Kettunen and L. Turner, "A volume integral formulation for nonlinear magnetostatics and eddy currents using edge elements," IEEE Trans. Magn., vol. 28, no. March, pp. 1639-1642, 1992.

[3] O. Chubar, P. Elleaume, J. Chavanne, "A three-dimensional magnetostatic computer code for insertion devices", J. Synchrotron Rad. Vol. 5, pp 481-484, 1998.

[4] M. Souza, C. Vidigal, A. Momy, J. Taquin, M. Sauzade, "Nonlinear calculation of 3D static magnetic fields", IEEE Trans. Magn, Vol 33. No 4, p2486-2491, July 1997.

[5] L. Greengard and V. Rokhlin, "A fast algorithm for particle simulations," J. Comput. Phys., vol. 73, no. 2, pp. 325-348, 1987.

[6] S. Borm and L. Grasedyck, "Hybrid cross approximation of integral operators," Numerische Mathematik, vol. 101, no. 2, pp. 221-249, Jun. 2005.

[7] J. C. Young and S. D. Gedney, "A Locally Corrected Nyström Formulation for the Magnetostatic Volume Integral Equation," IEEE Trans. Magn., vol. 47, no. 9, pp. 2163-2170, 2011.

[8] J. C. Young, S. D. Gedney, and R. J. Adams, "Quasi-Mixed-Order Prism Basis Functions for Nyström-Based Volume Integral Equations," IEEE Trans. Magn., vol. 48, no. 10, pp. 2560-2566, 2012.

[9] R. F. Harrington, "Field computation by moment methods", IEEE Press, April 1993.

[10] O. Chadebec, J.-L. Coulomb, and F. Janet, "A review of magnetostatic moment method," IEEE Trans. Magn., vol. 42, no. 4, pp. 515-520, Apr. 2006.

[11] Y. Takahashi, C. Matsumoto, and S. Wakao, "Large-Scale and Fast Nonlinear Magnetostatic Field Analysis by the Magnetic Moment Method With the Adaptive Cross Approximation," IEEE Trans. Magn., vol. 43, no. 4, pp. 1277-1280, Apr. 2007.

[12] Forsman, K., Kettunen, L., \& Nuutinen, J. P. "Properties of b-and h-type integral equation formulations," IEEE Trans. Magn., vol. 32, no. 3, pp. 1421-1424, 1996.

[13] L. Han, L. Tong, and J. Yang, "Integral equation method using total scalar potential for the simulation of linear or nonlinear 3D magnetostatic field with open boundary," IEEE Trans. Magn., vol. 30, no. 5, pp. 2897-2890, 1994.

[14] A. Carpentier, O. Chadebec, N. Galopin, G. Meunier and B. Bannwarth, "Resolution of Nonlinear Magnetostatic Problems With a Volume Integral Method Using the Magnetic Scalar Potential," IEEE Trans. Magn., vol. 49, no. 5, pp. 1685-1688, 2013.

[15] A. G. Kalimov, "Three-Dimensional Magnetostatic Field Calculation Using Integro-Differential Equation for Scalar Potential," IEEE Trans. Magn., vol. 32, no. 3, pp. 667-670, Mai 1996.

[16] A. Canova and M. Repetto, "Integral solution of nonlinear magnetostatic field problems," IEEE Trans. Magn., vol. 37, no. 3, pp. 1070-1077, May 2001.

[17] W. Hafla, F. Groh, A. Buchau, and W. M. Rucker, "Magnetic field computation with integral equation method and energy-controlled relaxation," IEEE Trans. Magn., vol. 42, no. 4, pp. 719-722, Apr. 2006.

[18] A. M. Vishnevsky, A. G. Kalimov, and A. A. Lapovok, "Modeling magnetization using Whitney facet elements," IEEE Trans. Magn., vol. 38, no. 2, pp. 489-492, Mar. 2002.

[19] S. Balasubramanian, S.N. Lalgudi and B. Shanker, "Fast-integralequation scheme for computing magnetostatic fields in nonlinear media", IEEE Trans.Magn., vol. 38, no. 5, pp. 3426-3432, 2002.

[20] A. Bossavit, "Whitney forms: a class of finite elements for threedimensional computations in electromagnetism," IEE Proc. A Phys. Sci. Meas. Instrumentation, Manag. Educ. Rev., vol. 135, no. 8, p. 493, 1988.

[21] A. Demenko, J.K. Sykulski "Geometric formulation of edge end nodal finite element equations in electromagnetics", The International Journal 
for Computation and Mathematics in Electrical and Electronic Engineering, Vol. 31, No. 5, 2012, pp. 1347-1357

[22] C. Giuffrida, G. Gruosso, and M. Repetto, "Finite formulation of nonlinear magnetostatics with integral boundary conditions", IEEE Trans. on Magnetics, Vol. 42, No. 5, pp. 1503 - 1511, May 2006

[23] C. J. Carpenter, "Finite-element network models and their application to eddy-current problems," vol. 122, no. 4, pp. 455-462, 1975.

[24] M. Gimignani, A. Musolino, and M. Raugi "Integral formulation for nonlinear magnetostatic and eddy currents analysis,", IEEE Trans. Magn, vol. 30, no. 5, pp. 3024-3027, 1994.

[25] T.-T. Nguyen, G. Meunier, J.-M. Guichon, O. Chadebec, and T.-S Nguyen, "An Integral Formulation for the Computation of 3-D Eddy Current Using Facet Elements," IEEE Trans. Magn., vol. 50, no. 2, pp. 549-552, 2014

[26] C. Rubeck, J. Yonnet, H. Allag, B. Delinchant, and O. Chadebec, "Analytical Calculation of Magnet Systems : Magnetic Field Created by Charged Triangles and Polyhedra," vol. 49, no. 1, pp. 144-147, 2013.

[27] T.-S. Nguyen, J.-M. Guichon, O. Chadebec, G. Meunier and B. Vincent, "An independent loops search algorithm for solving inductive PEEC large problems," Progress In Electromagnetics Research M, vol. 23, no. November 2011, pp. 53-63, 2012.

[28] T. Nakata, N. Takahashi, K. Fujiwara, "Summary of results for TEAM workshop problem 13 (3-D nonlinear magnetostatic model)", COMPEL vol. 14 Iss: 2/3, pp.91 - 101, 1995.

[29] Y. Saad, "A flexible inner-outer preconditioned GMRES algorithm", SIAM J. Sci. Comput. 14, pp. 461-469, 1993

[30] M. Bebendorf, "Appfroximation of boundary element matrices," Numer. Math., vol. 86, no. 4, pp. 565-589, Jun. 2000

[31] T.-S. Nguyen, J.-M. Guichon, O. Chadebec, P. Labie, and J.-L. Coulomb, "Ships magnetic anomaly computation with integral equation and fast multipole method," IEEE Trans. Mag., vol. 47, no. 5, pp. 1414-1417, May 2011

[32] V. Le-Van, B. Bannwarth, A. Carpentier, O. Chadebec, J.M. Guichon, and G. Meunier, "The Adaptive Cross Approximation Technique for a Nonlinear Magnetostatic Problems," IEEE Trans. Magn, vol. 50, no. 2, pp. 445 - 448, Feb. 2014. 